\title{
Concentration dependent interdiffusion in InGaAs/GaAs as evidenced by high resolution $x$-ray diffraction and photoluminescence spectroscopy
}

\author{
F. Bollet and W. P. Gillin ${ }^{\text {a) }}$ \\ Department of Physics, Queen Mary, University of London, Mile End Road, London, E1 4NS, \\ United Kingdom \\ M. Hopkinson \\ Department of Electronic Engineering, University of Sheffield, Mappin Street, Sheffield, S1 3JD, \\ United Kingdom \\ R. Gwilliam \\ Surrey Ion Beam Centre, Advanced Technology Institute, University of Surrey, Guildford, Surrey, \\ GU2 7XH, United Kingdom
}

(Received 16 June 2004; accepted 1 October 2004; published online 16 December 2004)

\begin{abstract}
A high resolution x-ray diffraction (HRXRD) and photoluminescence study of a $10 \mathrm{~nm}$ InGaAs/GaAs quantum well structure repeatedly diffused under thermally accurate and timed annealing conditions demonstrates that the Fickian model with a constant coefficient of diffusion is inadequate and that the distribution of compositions of the diffused well cannot be fitted with error functions. A simple model, with the well retaining its square shape and homogeneity while dissolving the barriers when annealed, is successful in modelling both the HRXRD and photoluminescence data. (C) 2005 American Institute of Physics. [DOI: 10.1063/1.1825613]
\end{abstract}

\section{INTRODUCTION}

There has long been an interest in the diffusion of III-V semiconductors. ${ }^{1}$ Because diffusion causes spatial variations in compositions, intermixing of heterostructures can alter their lattice parameter and the band gap energy. This diffusion can be undesirable when a device requires an abrupt change of composition, or it can be locally encouraged for design purposes by an appropriate capping or ion implantation to generate complex three-dimensional patterning.

A pseudomorphic crystalline quantum well is an ideal system to monitor diffusion as wells can be accurately grown and small diffusion lengths significantly alter their physical properties. Simple photoluminescence measurements, as a function of the annealing time of a quantum well, show changes in the recombination energy of excitons. These shifts in the photoluminescence energy are the direct result of the diffusion. By measuring how the photoluminescence of a single quantum well varies with annealing and by modeling this shift, using a simple Fickian diffusion model with a concentration independent diffusion coefficient, we have been able to determine the diffusion coefficient over a wide temperature range and consequently measure the activation energy for diffusion. ${ }^{2}$ Although this modeling technique has been widely used, our group pioneered the use of repetitive annealing of a single sample as a means of demonstrating the validity of the model through the linear variation of the measured diffusion length squared against anneal time. Despite this, there have been reports in the literature that have suggested that the diffusion might be concentration or strain dependant. ${ }^{1,3,4}$ It is also true that departure from the above

${ }^{a)}$ Electronic mail: w.gillin@qmul.ac.uk linear variation can be difficult to detect because the nonlinearity is not always dramatic ${ }^{5}$ and also because of a lack of data or scatter in the data points.

High resolution $\mathrm{x}$-ray diffraction (HRXRD) can offer a description of low-dimensional semiconductor structures and the distribution of diffusing species, ${ }^{6-9}$ especially in simple systems. InGaAs/GaAs is such a system as the extent and intensity of the strained domain generated by the distribution of indium in the specimen can be accurately measured. The repeated annealing of a single quantum well (SQW) specimen, coupled with photoluminescence and HRXRD measurements, shows beyond doubt that the Fickian model with a constant diffusion coefficient is inadequate, and that a simple square well model provides a better fit to all the experimental data. The success of the square well model implies that diffusion is occurring at a much higher rate in the well than in the barriers, allowing the well to retain a relatively square shape while dissolving the barriers. We have demonstrated that the model used to fit the HRXRD data is also consistent with the photoluminescence data.

\section{EXPERIMENTAL METHOD}

A SQW InGaAs/GaAs specimen was grown by molecular beam epitaxy. A GaAs buffer layer was first grown on a GaAs substrate, followed by a nominal $10 \mathrm{~nm} \operatorname{In}_{0.2} \mathrm{Ga}_{0.8} \mathrm{As}$ well layer and finally a $100 \mathrm{~nm}$ GaAs barrier. HRXRD measurements of the sample gave a thickness of $9.8 \mathrm{~nm}$ and a concentration of indium in the well was $19.2 \%$. The wafer was then capped with a $50 \mathrm{~nm}$ silicon nitride layer deposited by plasma enhanced chemical vapor deposition to prevent surface decomposition during annealing. A $5 \times 7 \mathrm{~mm}$ specimen cut from the wafer was then repeatedly annealed at $800{ }^{\circ} \mathrm{C}$ in helium ambient in a triple graphite strip rapid thermal annealer (rta) equipped with an Accufiber optical 


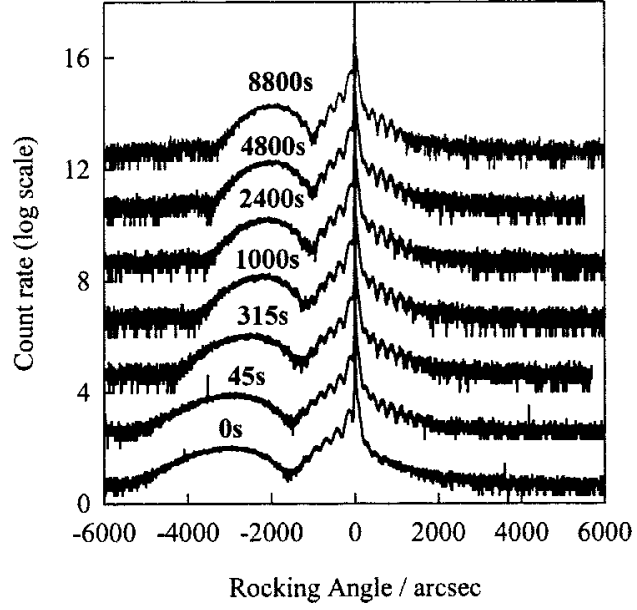

FIG. 1. Experimental 004 HRXRD rocking curves of an InGaAs/GaAs SQW annealed at $800{ }^{\circ} \mathrm{C}$. Annealing durations are indicated in seconds for each profile.

thermometry system for temperature control. The system is calibrated against the melting point of silver and is consistently accurate to $\pm 1{ }^{\circ} \mathrm{C}$. A linear ramp time of $15 \mathrm{~s}$ was used to reach the anneal temperature.

After each annealing step the photoluminescence energy and 004 HRXRD profiles of the specimen were measured. The photoluminescence spectra were collected from the specimen cooled to $80 \mathrm{~K}$ and excited with the $514.5 \mathrm{~nm}$ line of an argon ion laser. 004 HRXRD rocking curves were measured at room temperature on a Philips material research diffractometer (MRD). The MRD had a highly collimated monochromatic x-ray beam from the $\mathrm{Cu} K \alpha$ radiation produced using the 220 reflections of a four-crystal Bartels germanium monochromator. The Philips X'PERT EPYTAXY 3.0 software allowed the calculation of rocking curves for various virtual crystals made of layers of uniform composition using the dynamical theory of x-ray scattering. Its companion software SMOOTHFIT, allowed the curve fitting of the experimental measurements.

\section{RESULTS}

Figure 1 shows the 004 HRXRD rocking curves for the InGaAs/GaAs sample following annealing at $800{ }^{\circ} \mathrm{C}$ for various times. The broad peak, characteristic of the quantum well layer, can be seen migrating toward the substrate peak and getting thinner with increasing annealing time. It indicates that, as expected, with the diffusion of indium, the strain in the quantum well layer decreases and the well itself broadens. The small Pendellösung fringes visible around the substrate peak are characteristic of the decoupling introduced by the quantum layer between the x-ray coherent reflections from the GaAs substrate/buffer layer and the top barrier layer. The periodicity of the fringes is related to the thickness of the top GaAs barrier layer.

Some diffusion profiles of indium were calculated for various diffusion lengths by assuming a Fickian diffusion with a constant diffusion coefficient and using the error function approach. The Philips X'PERT EPITAXY 3.0 software could then simulate the corresponding 004 rocking curves. Figure 2

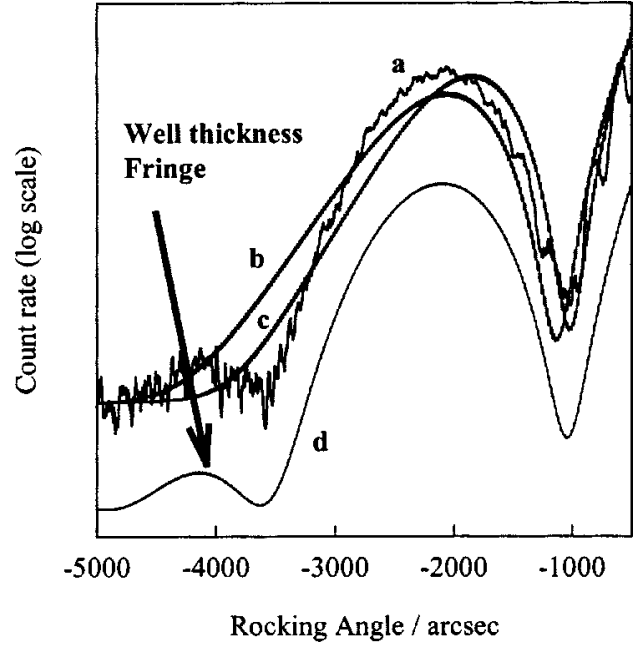

FIG. 2. (a) Smoothed experimental 004 HRXRD rocking curves for the InGaAs/GaAs SQW annealed $2400 \mathrm{~s}$ at $800^{\circ} \mathrm{C}$, (b) and (c) are simulations generated by composition distributions calculated with error functions, and (d): $14.5 \mathrm{~nm} \operatorname{In}_{12.9} \mathrm{Ga}_{87.1}$ As square well.

displays the experimental rocking curve for the specimen annealed $2400 \mathrm{~s}$ at $800{ }^{\circ} \mathrm{C}$ and superimposed the simulated Fickian profiles for diffusion lengths of 5 and $6 \mathrm{~nm}$, the latter having a calculated photoluminescence peak shift very close to the experimental value. These simulated rocking curves bound the front of the quantum well peak between $\sim-1000$ and $\sim-2000$ arcsec and, clearly, the experimental curve clearly cannot be fitted with the error function profiles. The experimental data is sharper with a second feature at -4200 arcsec. A much closer fit is obtained with a purely square model, as seen in Fig. 2 with the translated rocking curve simulation. This fitting profile was generated by simulating a $14.5 \mathrm{~nm}$ homogeneous square well containing $12.90 \%$ indium buried under a GaAs barrier. It is an extreme approximation of a situation where diffusion in the well layer occurs at a much higher rate than in the barriers, the latter being effectively dissolved by a well that retains a square shape and homogeneous composition.

Each experimental rocking curve was fitted using the SMOOTHFIT software with a homogeneous square well profile by leaving the well width and composition as free fitting parameters. Figure 3 shows several fits at different stages of annealing. The total amount of simulated indium remained consistently close to the value deduced from fitting the unannealed specimen HRXRD rocking curve. A comparison of the total indium (the thickness concentration product) in the unannealed well and each of the annealed wells shows a maximum variation of $\sim 1 \%$.

The photoluminescence energies of the square well simulations were then calculated and compared to the experimental measurements. Figure 4 shows a very good agreement between calculated and experimental values of the photoluminescence shift with $\mathrm{In} / \mathrm{Ga}$ interdiffusion. The calculated photoluminescence energies were slightly larger than the experimental data with a systematic error of about $15 \mathrm{meV}$. This is a relatively small value and the fact that it is a systematic error suggests that it could be accounted for by the exciton binding energy, the temperature at which the em- 


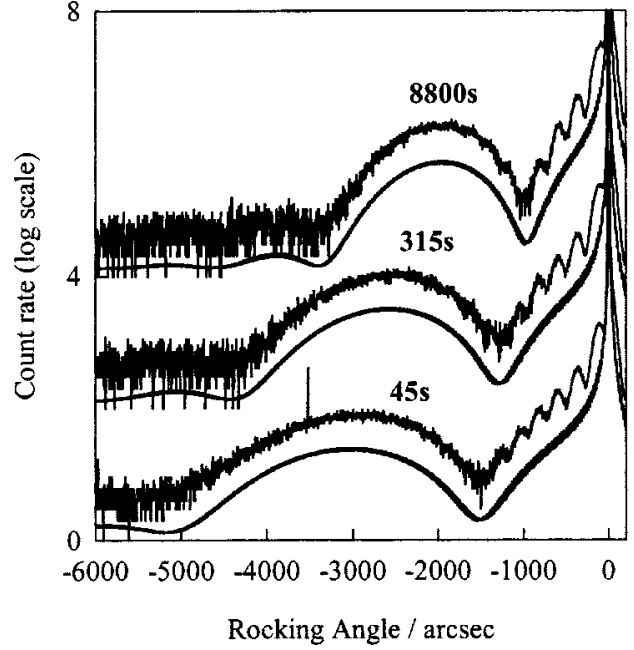

FIG. 3. Experimental 004 HRXRD rocking curves of the InGaAs/GaAs SQW at various stages of annealing at $800{ }^{\circ} \mathrm{C}$. Annealing durations are indicated in seconds for each profile. The square well model fits are from bottom to top: $10.2 \mathrm{~nm} \mathrm{In}_{18.6} \mathrm{Ga}_{81.4} \mathrm{As}, 11.9 \mathrm{~nm} \mathrm{In}{ }_{15.8} \mathrm{Ga}_{84.2} \mathrm{As}$, and $15.4 \mathrm{~nm}$ $\mathrm{In}_{12,1} \mathrm{Ga}_{87,9} \mathrm{As}$

piric formulas used for the energy level calculations in the InGaAs/GaAs system was measured, or the approximation that the well is perfectly homogeneous in composition.

\section{DISCUSSION}

Figures 5 and 6 are calculated models displaying the photoluminescence peak shift as a function of diffusion length for $\mathrm{In}_{0.18} \mathrm{Ga}_{0.82} \mathrm{As} / \mathrm{GaAs}$ quantum wells of various nominal widths. Figure 5 refers to the Fickian model with a constant coefficient of diffusion. Figure 6 is the square well model in which the well dissolves the barriers, retains a square shape, and a homogeneous composition with diffusion. The diffusion length is defined as half the total well width increase, and the well composition is calculated by a simple conservation of matter with

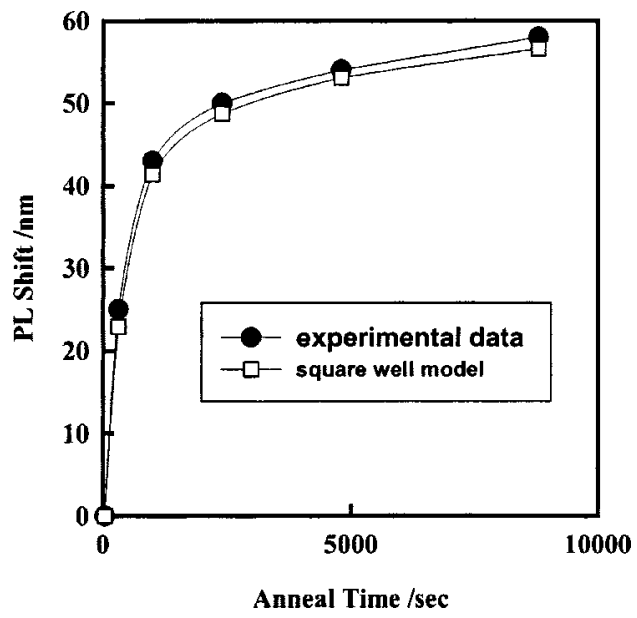

FIG. 4. The empty squares are the experimental photoluminescence shift vs anneal times at $800{ }^{\circ} \mathrm{C}$ of the InGaAs SQW. The solid squares are the values calculated for the square wells that fit the experimental HRXRD curves in Figs. 1-3.

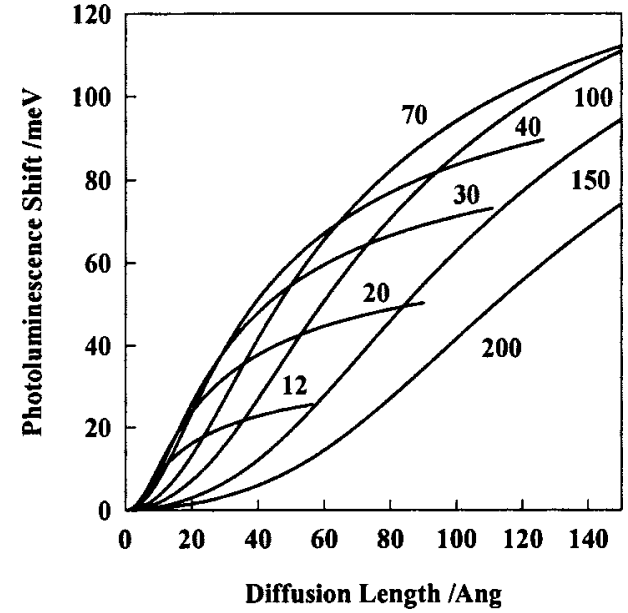

FIG. 5. Simulations of photoluminescence shift vs diffusion length for $\mathrm{In}_{18} \mathrm{Ga}_{82}$ As SQWs of various nominal widths using a Fickian model with a constant coefficient of diffusion. The nominal widths are indicated in Angström on each curve.

$$
\operatorname{In}_{W}=\frac{\operatorname{In}_{0} \times w_{0}}{w},
$$

where $\operatorname{In}_{W}$ is the concentration of indium in a diffused well of width $w$, and $\operatorname{In}_{0}, w_{0}$ are the indium concentration and width of the undiffused well.

The occurrence of a quantum well thickness fringe in the experimental profile displayed in Fig. 2 is strong evidence that the well is retaining a square shape even after a long annealing time. As an approximation, the rocking curve measured in reciprocal space is a Fourier transform of the specimen structure in real space. A progressive loss of abruptness between the heterogeneous layers of the specimen would render the higher order terms in a Fourier series negligible, which would be observed as an attenuation of higher order fringes in the rocking curve. The origin of Pendellösung fringes generated by a family of diffracting planes is nevertheless more complex than for equal thickness fringes ob-

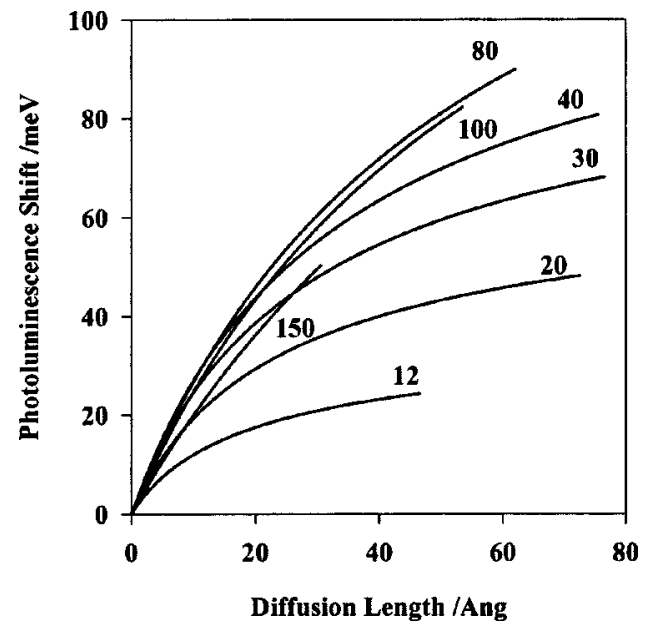

FIG. 6. Simulations of photoluminescence shift vs diffusion length for $\mathrm{In}_{18} \mathrm{Ga}_{82} \mathrm{As}$ SQWs of various nominal widths using a simple square well model where the well retains a square shape and homogeneous composition with diffusion. The nominal widths are indicated in Angström on each curve. 
served in the simple Fraunhofer case in reflectivity measurements. It makes direct interpretation of rocking curves difficult. For example, for long x-ray coherence length, diffraction for small layers and the substrate cannot be treated independently of each other. A direct result of this, is that a thin layer peak neighboring the intense substrate peak is distorted and displaced. For this reason, the modeling of the rocking curve by a dedicated software using the generally accurate dynamical calculation for $\mathrm{x}$-ray scattering is currently the most reliable method to extract structural information. ${ }^{8}$ Fringes are not visible in the case of graded interfaces and cannot be seen in the simulated rocking curves calculated with the Fickian model (Fig. 2). The square well hypothesis is successful in modeling the experimental HRXRD rocking curves and the experimental photoluminescence peak shift with diffusion. Furthermore, the distribution of compositions calculated with error functions is in clear disagreement in Fig. 2 with our experimental HRXRD measurements.

From these results, it is obvious that it cannot be assumed that a Fickian model with a constant coefficient of diffusion will provide meaningful information for diffusion experiments in the InGaAs/GaAs system. A closer look at Figs. 5 and 6 shows that fitting experimental data with either model can yield very different or even contradictory answers to a problem. An example is experiments investigating possible vacancy injection or destruction in some layers of a heterostructure by monitoring the photoluminescence peak shift of diffusing wells of different widths that have been grown at various depths in a specimen. The apparent dependence of the diffusion rates with wells depth has often been concomitant with the broader wells diffusing faster, as seen in Refs. 10 and 11, for example. This is certainly an artifact resulting from using a wrong model to fit experimental data. Using the Fickian assumption with a constant coefficient of diffusion to model experimental data of various experiments conducted in our group consistently yields the apparent results of broader wells of identical compositions diffusing faster under identical annealing conditions. A trend with well composition in the InGaAs/GaAs system is also noticed.

\section{CONCLUSIONS}

HRXRD is a powerful tool that can provide accurate information on the composition distribution of small diffused quantum wells. The method is sensitive enough to conclude that in our experiments, for the InGaAs/GaAs system, interdiffusion under the effect of annealing cannot be assumed to be following Fick's laws with a constant coefficient of diffusion, and that the modeling of diffused composition profiles with error functions is wrong. InGaAs/GaAs is a simple system in which there is no miscibility gap and indium simply interdiffused with gallium. The failure of the above model for this system therefore implies that methods of quantifying diffusion in other semiconductor structures that rely solely on measuring the photoluminescence peak shift can lead to erroneous conclusions.

${ }^{1}$ L. L. Chang and A. Koma, Appl. Phys. Lett. 29, 138 (1976).

${ }^{2}$ S. W. Wee, M. K. Chai, K. P. Homewood, and W. P. Gillin, J. Appl. Phys. 82, 4842 (1997).

${ }^{3}$ H. Temkin, S. N. G. Chu, M. B. Panish, and R. A. Logan, Appl. Phys. Lett. 50, 956 (1987).

${ }^{4}$ F. Bollet, W. P. Gillin, M. Hopkinson, and R. Gwilliam, J. Appl. Phys. 93, 3881 (2003)

${ }^{5}$ W. P. Gillin and D. J. Dunstan, Phys. Rev. B 50, 7495 (1994).

${ }^{6}$ F. Bollet and W. P. Gillin, J. Appl. Phys. 94, 988 (2003).

${ }^{7}$ L. Tapfer and K. Ploog, Phys. Rev. B 40, 9802 (1989).

${ }^{8}$ P. F. Fewster, Semicond. Sci. Technol. 8, 1915 (1993).

${ }^{9}$ C. Bocchi and C. Ferrari, J. Phys. D 28, A165 (1995).

${ }^{10}$ W. P. Gillin, D. J. Dunstan, K. P. Homewood, L. K. Howard, and B. J. Sealy, J. Appl. Phys. 73, 3782 (1993).

${ }^{11}$ W. J. Taylor, N. Kuwata, I. Yoshida, T. Katsuyama, and H. Hayashi, J. Appl. Phys. 73, 8653 (1993). 